\title{
The peak region of the extragalactic background radiation
}

\author{
Marco Ajello*i \\ Stanford Linear Accelerator Center, 2575 Sand Hill Road, Menlo Park, CA 94025 and \\ KIPAC, 2575 Sand Hill Road, Menlo Park, CA 94025 \\ E-mail: majello@slac.stanford.edu
}

The Cosmic X-ray background carries the information of cosmic accretion onto super-massive black holes. The intensity at its peak can be used to constrain the integrated space density of highly obscured AGNs. Determining the shape and intensity of the Cosmic X-ray background radiation represents, however, a first step towards the understanding of the population of Comptonthick AGNs. The study of AGNs in the local and more distant Universe allows to understand the whole picture. In this talk, I will review the current understanding of generation of the Cosmic $\mathrm{X}$-ray background at its peak. I will focus on the recent measurements of the Cosmic X-ray background and I will discuss the recent advancements in the understanding of AGNs in both the local and more distant Universe. Finally, I will also discuss open issues and future prospects.

7th INTEGRAL Workshop

September 8-112008

Copenhagen, Denmark

* Speaker.

${ }^{\dagger}$ I thank the organizers for the invitation to give this talk and for organizing a scientifically stimulating event. 


\section{Introduction}

\subsection{History of the Cosmic X-ray background}

The discovery of the cosmic X-ray background (CXB) happened at the same time as the detection of the very first extra-solar X-ray source (1). Both of them marked the beginning of a new era in high-energy astrophysics. Since then, the CXB background has been the object of a lively debate. First about its origin, later about its spectral shape. Indeed, a diffuse isotropic radiation such as the CXB might be produced either by hot gas permeating the Universe, or by millions of point-like X-ray sources or by both. Precise measurements done with the HEAO1-A2 experiment (2) revealed that the CXB spectral shape is consistent, between 3-50 keV with a bremmstrahlung model with a temperature of $40 \mathrm{keV}$. This was seen as a natural evidence for the presence of a very hot intergalactic medium. This conclusion was supported by, or was based on, the power-law like emission of Active Galactic Nuclei (AGNs, e.g. 3), whose integrated emission (in the case millions of AGNs existed), remains always a power-law like spectrum. The final resolution of the controversy came from the incredibly neat result obtained with the FIRAS instrument on board COBE: the absence of any detectable deviation from a pure black spectrum body of the cosmic microwave background set an upper limit on the contribution of an uniform hot intergalactic gas to the CXB of $<10^{-4}$ (4). Once this issue was solved, it was clear that the CXB emission had to be the unresolved (at that time) emission of millions of AGNs (5). The deep X-ray surveys $(6 ; 7 ; 8)$ have confirmed that indeed a large fraction (80-100\%) of the CXB can be resolved into point-like sources which can then be identified as AGNs. The discovery that for many AGNs the nuclear radiation is partially obscured by intervening matter led population synthesis models to solve the paradigm of the generation of the CXB $(9 ; 10 ; 11 ; 12)$ by means of AGNs with different amount of absorption. Although the Chandra and XMM-Newton have resolved most of the Cosmic X-ray background below $\sim 2 \mathrm{keV}$, the fraction of resolved CXB emission declines with energy being $<50 \%$ above $6 \mathrm{keV}$ (13). This represents the main evidence for the presence of a population of AGNs which is still currently undetected even in the deepest surveys. The analysis of the unresolved component revealed that it might be consistent with the integrated emission of a population of very absorbed, Compton-thick ( $\tau=N_{H} \sigma_{T} \sim 1$ and thus $N_{H} \approx 1.5 \times 10^{24}$ atoms $\mathrm{cm}^{-2}$ ) AGNs. Given the fact that their emission is suppressed below $10 \mathrm{keV}$, detecting these object is extremely difficult at soft $\mathrm{X}$-rays and until a few years ago only a handful of Compton-thick AGNs were known (14).

\subsection{Constraints from the Cosmic X-ray background}

Since the direct detection of Compton-thick objects is difficult in X-rays, the CXB spectrum becomes the final resource to constrain the space density of such objects. Indeed, the CXB represents the integrated emission of the accretion processes onto super-massive black holes (SMBHs) present in the Universe. Integrating the luminosity function of unabsorbed and absorbed AGNs $(15 ; 10)$, with sensible hypotheses regarding their spectral properties (and their dispersions), allows an estimate of the contribution of these two classes to the total CXB spectrum. The most recent studies $(10 ; 11 ; 12)$ show that the contribution of these two classes is not enough to explain the totality of the CXB highlighting a deficit around the CXB peak at $30 \mathrm{keV}$. Assuming that this deficit emission is due to undetected Compton-thick AGNs, it becomes possible to make an estimate of their space density. With no other constrains left, except the small number of heavily 
obscured objects known in the local Universe, the absolute normalization of the CXB spectrum (particularly at its peak) represents the main resource to constrain the Compton-thick population. This is why it has been much debated lately. In the $2-10 \mathrm{keV}$ band the CXB measurements of focusing telescopes (as XMM-Newton, Chandra, etc.) lie systematically above the one obtained by non-focusing optics (16). Moreover, it seems that neither cosmic variance (17) nor differences in the flux scale calibration of each individual instrument $(16 ; 18)$ may account for this discrepancy. This led several authors to naturally question the broadband measurement of the CXB performed by HEAO1 (19) and to use CXB spectra renormalized by a factor $\sim 1.3(10 ; 11 ; 13)$. A change in the normalization of the CXB implies either a change in the number density of AGNs or in their radiative efficiency or in both. This is why the normalization of the CXB has been so much debated in the past. In particular the lack of measurements at the peak of the CXB $(\sim 30 \mathrm{keV})$ left the population of Compton-thick AGNs loosely constrained. This situation recently improved with the results shown in the next sections.

\section{Advances in the measurement of the Cosmic X-ray background}

Measuring the $\mathrm{CXB}$ is extremely challenging with currently flying instruments as none of them has been directly designed to make such measurement. The main difficulty here is the capability, by design or analysis, to discriminate the CXB among the other, sometimes dominating, instrumental background components. In the case of INTEGRAL and Swift, it is even worst as their high-energy detectors (IBIS and BAT $(20 ; 21)$ ) are coded-mask detectors expressly designed for the study of point-like sources. Measurements of the CXB have thus to rely on indirect techniques as the one based on the modulation of the CXB signal by the passage of the earth in the field of view (FOV). All the recent measurements performed by INTEGRAL, BeppoSAX and Swift/BAT $(22 ; 18 ; 23)$ use the earth occultation technique. These measurements, see Fig. 1, are more consistent with the original HEAO1 CXB spectrum rather than with its renormalization by $\sim 1.3$ (see discussion in the previous section). The INTEGRAL and Swift/BAT CXB spectra lie $\sim 10 \%$ above the HEAO1 spectrum. Unfortunately, the albedo emission from the earth, which must be taken into account during the occultation analysis, does not allow to make a strong test of the shape of the CXB spectrum. This test becomes only possible when different measurements and instruments are used. Taking into account all the newest measurements and neglecting the broadband measurement of HEAO1 it is possible to show that a simple description of the CXB spectrum is achieved using a smoothly joined double power-law function (23). From this analysis, it appears that the CXB spectrum, as measured most recently is $\sim 30 \%$ larger than the HEAO1 spectrum below $10 \mathrm{keV}$ while only $10 \%$ larger than it above this energy. A new measurement performed in the $1-7 \mathrm{keV}$ band with the X-ray telescope (XRT), on board Swift, confirms this picture (24).

\section{Hard X-ray surveys}

The understanding of AGN activity, growth and evolution can be achieved by combining shallow large area sky surveys to very deep pencil beam surveys $(15 ; 25 ; 26)$. Since most of the AGNs are absorbed (e.g. (12)) the best method to select a representative sample of the entire AGN population is to use an energy band which is the least affected by attenuation due to intrinsic absorption. 


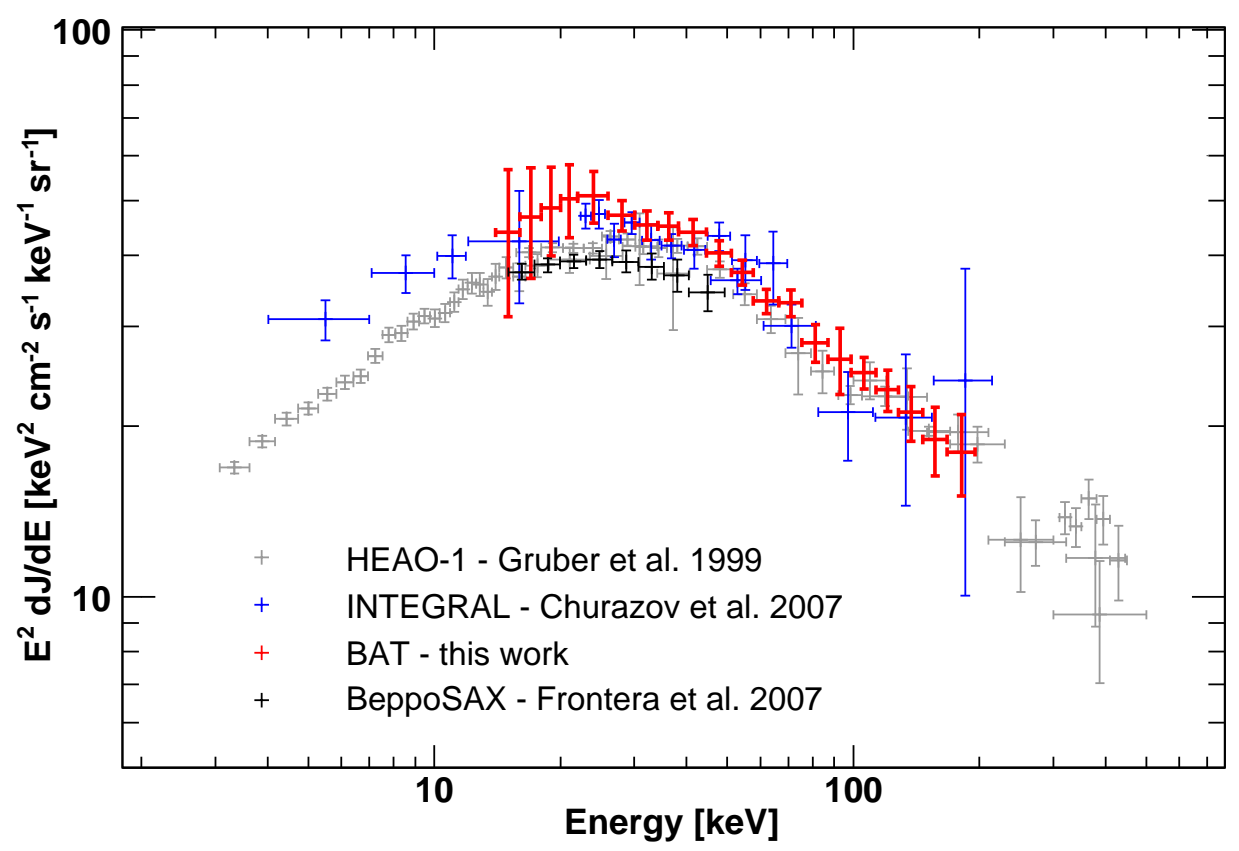

Figure 1: Comparison of measurements of the peak of the Cosmic X-ray background (adapted from (23)).

As of now, this is the $2-10 \mathrm{keV}$ energy band because above this energy no deep surveys exist. Recently, (27) compiled the largest set of X-ray surveys in the $2-10 \mathrm{keV}$ band. According to Hasinger 2008, to best determine AGN evolution one should achieve a good sampling of the luminosityredshift plane. From Fig. 2 (left panel) it is evident that while Chandra and XMM-Newton are able to perform a good sampling of the high-luminosity high-redshift part of the plane, the sampling remain sparse at low luminosities and low redshifts lacking a large sample able to constrain the properties of AGNs in the local Universe. Conversely, the $>10 \mathrm{keV}$ range lacks deep surveys (able to sample fluxes of $10^{-13}-10^{-14} \mathrm{erg} \mathrm{cm}^{-2} \mathrm{~s}^{-1}$ ), but on the other hand, the IBIS and BAT surveys (e.g. $28 ; 29 ; 30 ; 31 ; 32$ ) are detecting hundreds of AGNs in the local Universe. This is clearly shown in the right panel of Fig. 2. Moreover, as Fig. 3 shows, the $>15 \mathrm{keV}$ band is the best one for an unbiased (with respect to absorption) search of AGNs. Indeed, for absorbing column densities of $10^{23}$ and $10^{24}$ atoms $\mathrm{cm}^{-2}$ the fraction of nuclear flux which is detected in the $2-10 \mathrm{keV}$ band is $50 \%$ and $7 \%$ respectively. The $15-55 \mathrm{keV}$ band, as an example, is unbiased up to $\sim 10^{24}$ atoms $\mathrm{cm}^{-2}$. This is particularly important if the goal of the survey is the detection and understanding of the still missing Compton-thick source population.

\subsection{The role of absorption}

The AGN unified models predict that $\sim 3 / 4$ of all AGNs should be absorbed by intervening matter along the line of sight. This prediction is based on the observations of ionization cones, in $\mathrm{O}$ [III] light, with apex at the nucleus (e.g. 34). However, this is clearly not the case for the X-ray selected AGNs in the local Universe. Indeed, as Tab. 1 shows the fraction of absorbed AGNs is 

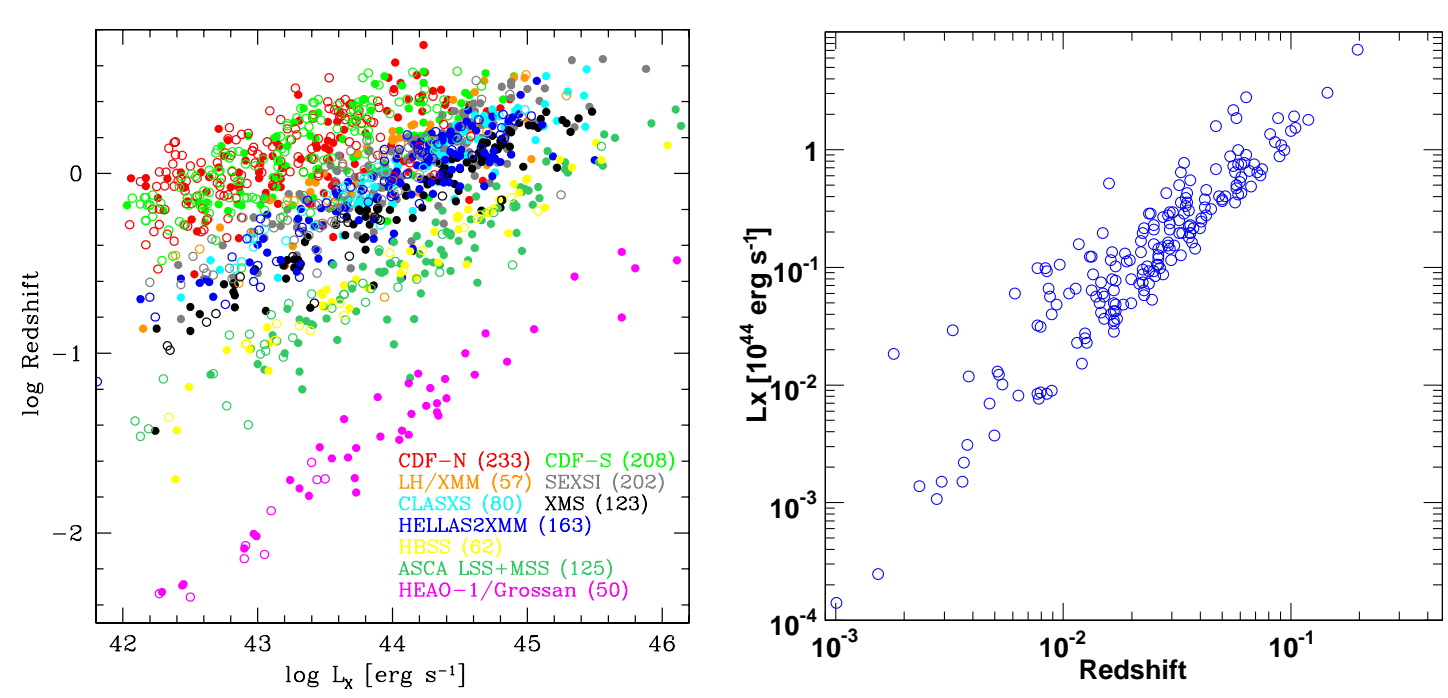

Figure 2: Left Panel: Meta-sample compiled by (27) using the available X-ray surveys in the $2-10 \mathrm{keV}$ energy band. Right Panel: BAT AGN sample $\left(|\mathrm{b}|>15^{\circ}\right)$ from $\sim 3$ years of all-sky survey in the $15-55 \mathrm{keV}$ band. INTEGRAL samples luminosities and redshifts similar to BAT (e.g. 28; 29; 30). A typical luminosity of $10^{44} \mathrm{erg} \mathrm{s}^{-1}$ in the $15-55 \mathrm{keV}$ is equivalent to $\sim 1.2 \times 10^{44} \mathrm{erg} \mathrm{s}^{-1}$ in the $2-10 \mathrm{keV}$ energy band.

significantly lower than $75 \%$ and closer to $\sim 50 \%$. Moreover, it is now well established that the fraction of obscured AGNs is not constant, but that it evolves with luminosity (e.g. 35; 10; 27). This yields that low-luminosity AGNs are surrounded by an obscuring medium which covers a large solid angle ( $80 \%$ of the sky as seen from the nucleus) while high-luminosity AGNs have a smaller covering factor and thus must be able to clean their environment. This effect can be interpreted in the framework of the receding torus model (e.g. 36) as the increase of the dust sublimation radius as a function of AGN luminosity. This would produce a decrease of the solid angle which the dusty torus covers around the nuclear source, explaining, qualitatively, the observed trend with luminosity. However, the receding torus model fails to explain the exact trend of the absorbed fraction with luminosity (37). Another option is that the torus is not formed by a continuous distribution of dust particles, but that it comprises of several optically thick clouds orbiting around the central source (e.g. 38; 39). In this framework, the torus consists of a large number of small, self-gravitating, dusty molecular clouds which form a clumpy medium (e.g. 40). This model explains the observed trend of the absorbed fraction with luminosity very well and this effect might be produced either by a change in the torus width or in the number of clouds or in both (see e.g. 39 , for details). In any case, the anti-correlation of luminosity and absorption has been interpreted as one of the main evidences for the breakdown of the AGN unified model.

\subsection{Compton-thick AGNs in the local Universe}

Compton-thick AGNs may come in two flavors: transmission-dominated and reflection-dominated sources (see e.g. 14). For trasmission-dominated objects, where a fraction of the nuclear emission (piercing through the Compton-thick torus) is detected, the spectral shape varies accordingly 


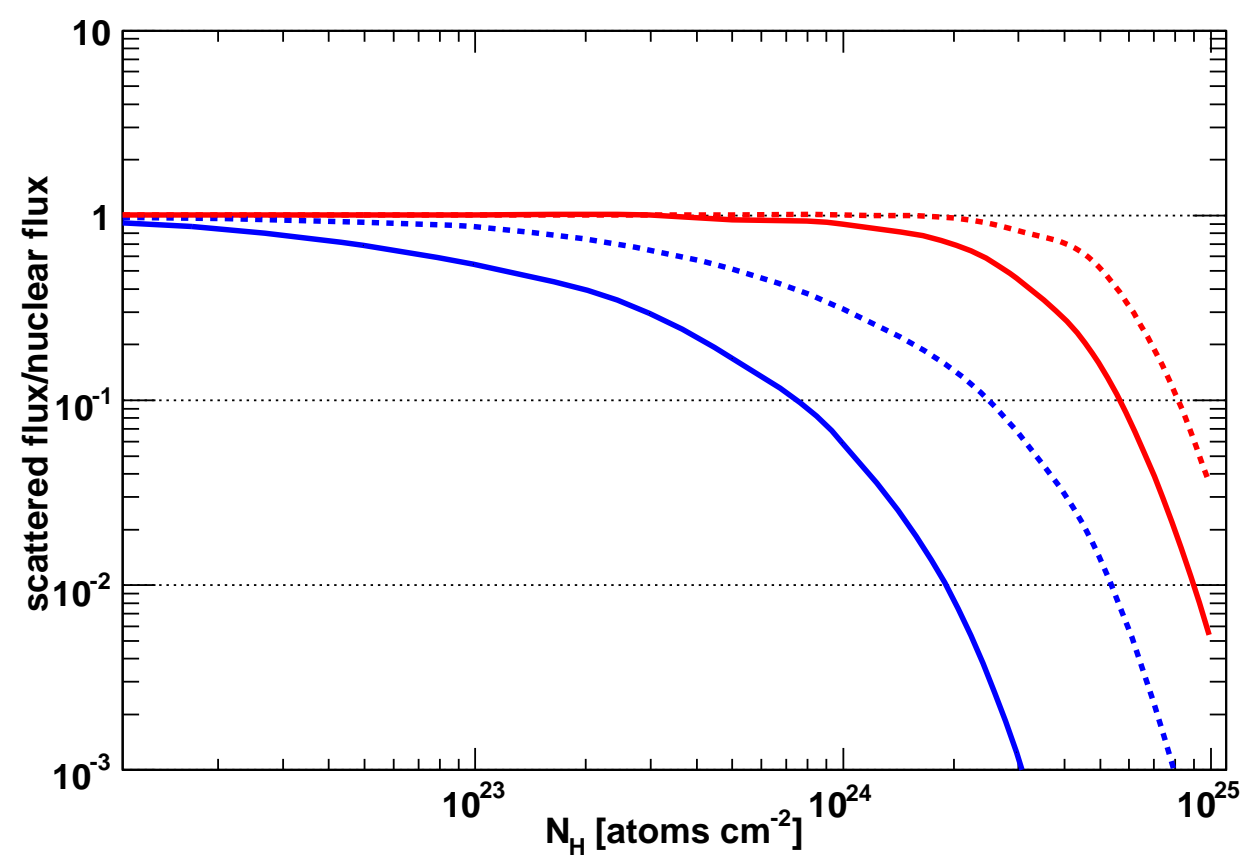

Figure 3: Ratio of observed flux (scattered) to intrinsic nuclear flux as a function of column density along the line sight for the $2-10 \mathrm{keV}$ (blue) and the $15-55 \mathrm{keV}$ (red) bands. The solid and dashed lines are for the case of an AGN at redshift 0 and 1 respectively. Compton scattering as well as photoelectric absorption has been taken into account using the model of (33). The nuclear emission has been modeled as a power law with photon index of 2.0 .

to the absorbing column density and the photoelectric cut-off moves progressively to higher energy. For increasing column densities $\left(24<\operatorname{LogN}_{H}<25\right)$, the absorbing material becomes more and more efficient in suppressing the radiation below $10 \mathrm{keV}$ and Compton recoil makes steeper (down-scattering) the $>10 \mathrm{keV}$ part of the spectrum. Transmission-dominated sources (also called heavily Compton-thick objects) usually show a broad iron $\mathrm{K}_{\alpha}$ line over a flat continuum and the ratio of the observed to the nuclear flux can be as low as a few \% (e.g. 41; 42). Studies of the local Universe in the Optical showed that Compton-thick AGNs should be as numerous as normally obscured ones (e.g. 43; 44), however up to now only a handful bona-fide Compton-thick objects were detected $(14 ; 45)$.

Results from the BAT and INTEGRAL surveys allow to shed some light on the overall picture. Tab. 1 shows an up to date status of hard X-ray surveys. It is apparent that at the fluxes currently sampled, the fraction of Compton-thick objects is bound to be $<20 \%$ and likely closer to $10 \%$. Strictly speaking this represents a lower limit on the real fraction since, as I showed in Fig. 3, even above $>15 \mathrm{keV}$ not all the source flux can be detected. This low number of Compton-thick AGNs seems, however, to be in agreement with the prediction of population synthesis models which require a substantial contribution from Compton-thick objects to explain the peak of the CXB emission (e.g. 12). In other words, Compton-thick AGNs seem to exist, but even INTEGRAL and 
BAT are not sensitive enough to detect many of them in the local Universe. If these sources evolve similarly to the other classes of AGNs (e.g. 15; 25), then their number density is expected to raise quickly with redshift peaking around $z \approx 1$. In this respect, instruments able to sample the $10^{-13}$ $10^{-14} \mathrm{erg} \mathrm{cm}^{-2} \mathrm{~s}^{-1}$ fluxes above $15 \mathrm{keV}$ have more chances to detect a large number of heavily absorbed objects. In this case the $k$-correction plays also in their favour allowing to sample the source spectrum at an higher energy as Fig. 3 shows.

Another interesting point is that the current hard X-ray surveys have not detected any new Compton-thick AGNs of the trasmission-dominated class. They have found many objects with very high column densities which are almost Compton-thick (e.g. $\mathrm{N}_{H} \approx 10^{23.8-24.0}$ atoms cm ${ }^{-2}$ ), but not completely so. Quoting (46), who analyzed the Tueller et al. (2008) sample,:If we take the Compton-thick definition to apply to sources whose column densities are $>1.4 \times 10^{24} \mathrm{~cm}^{-2}$, none of the BAT-detected sources are Compton-thick. This is also due to the fact that when fitting broad-band X-ray data (e.g. 0.1-200 keV), it is difficult (with the low signal-to-noise ratio at high energy) to discriminate between alternative interpretations. Indeed, most of the time reflection, partial absorbers and cut-off components remain degenerate.

Table 1: Fraction of absorbed and Compton-thick AGNs, relative to the whole population for different hard $\mathrm{X}$-ray surveys.

\begin{tabular}{lccccc}
\hline Reference & Completeness & \% Absorbed & $\%$ C-thick & $\begin{array}{c}\text { Band } \\
(\mathrm{keV})\end{array}$ & Instrument \\
\hline Markwardt et al., 2005 & $95 \%$ & $\sim 64 \%$ & $\sim 10 \%$ & $15-200$ & Swift/BAT \\
Beckmann et al., 2006 & $100 \%$ & $\sim 64 \%$ & $\sim 10 \%$ & $20-40$ & INTEGRAL \\
Bassani et al., 2006 & $77 \%$ & $\sim 65 \%$ & $\sim 14 \%$ & $20-100$ & INTEGRAL \\
Sazonov et al., 2007 & $90 \%$ & $\sim 50 \%$ & $\sim 10-15 \%$ & $17-60$ & INTEGRAL \\
Ajello et al., 2008a & $100 \%$ & $\sim 55 \%$ & $<20 \%$ & $14-170$ & Swift/BAT \\
Tueller et al., 2008 & $100 \%$ & $\sim 50 \%$ & $\sim 5 \%$ & $14-195$ & Swift/BAT \\
Paltani et al., 2008 & $100 \%$ & $\sim 60 \%$ & $<24 \%$ & $20-60$ & INTEGRAL \\
Della Ceca et al., 2008 & $97 \%$ & $\sim 57 \%$ & 0 & $4.5-7.5$ & XMM-Newton \\
\hline & & & & \\
&
\end{tabular}

However, a major discovery was achieved by INTEGRAL and Swift and this is the detection of "buried" super-massive black holes $(42 ; 41)$. These are AGNs for which the reflection component dominates over the transmitted one. If the ratio of the normalizations of these two components is interpreted as the solid angle covered by the reflecting medium (i.e. $\mathrm{R}=\Omega / 2 \pi$ ) then this value exceeds 1 . This would imply that part of the nuclear emission is blocked by nonuniform material along the line of sight even above $10 \mathrm{keV}$. The extremely low scattering efficiency which has been found for these objects (e.g. $0.5-2 \% 42 ; 41$ ) implies a torus half-opening angle of $<20^{\circ}$ in contrast to the classical $30^{\circ}-40^{\circ}$ expected in the framework of AGN unified model (e.g. 49). The large equivalent widths ( $\sim 1 \mathrm{keV}$, see Fig. 4$)$ of the iron $\mathrm{K}_{\alpha}$ line and the absence or weakness of [O III] lines confirm this interpretation. 
In order to recognize these objects as such, one needs sensitive and broad-band X-ray coverage. Thus it might well be that a few (or many) of these objects are hiding among the INTEGRAL and Swift/BAT survey sources. According to (46) these objects might be a relevant fraction, $\sim 25 \%$, of the total AGN population of the local Universe. The question whether this is the missing source population (i.e. the one needed to explain the totality of the CXB emission at its peak) is a difficult one to ask. The answer might come only from a sample of buried AGN large enough to derive its luminosity function. Until that time (37) derived the first luminosity function of Compton-thick AGNs as a difference between the Optical luminosity function of AGNs derived by (50) and their $\mathrm{X}$-ray luminosity function of AGNs. They find that the space density of Compton-thick objects is twice the density of Compton-thin AGNs with an hard upper limit to four times this density given by the limit imposed by the local black hole mass density (51).
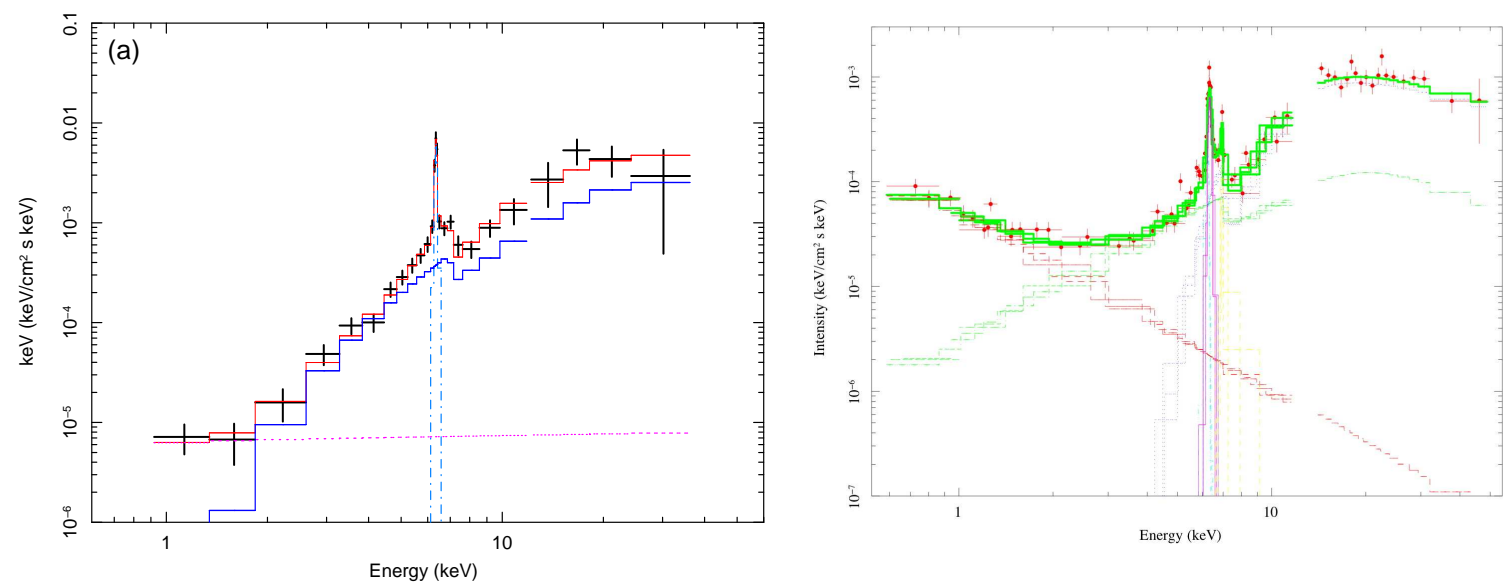

Figure 4: Example of Suzaku X-ray spectra of two buried AGNs. Note the dominating reflection component (which is curved at high energy) and the strong iron line. For both objects (SWIFT J0601.9-8636 and NGC 5728 left and right respectively) the scattering efficiency is very low (0.2-2\%). Adapted from (42) (left) and (41) (right).

\subsection{Compton-thick AGNs in the high-z Universe}

As already seen, selecting Compton-thick objects is extremely difficult even in hard X-rays. There is however a possibility to recover obscured AGNs thanks to the reprocessing of the AGN UV emission in the infrared (IR) band. Thus, selecting bright mid-IR sources which are faint in the Optical, and thus likely to be obscured, might be rewarding (e.g. 52; 53). Such window of opportunity has been recently opened by the Spitzer telescope. Indeed, using Spitzer (52) has found that bright mid-IR sources $(\mathrm{F}(24 \mu \mathrm{m})>0.3 \mathrm{mJy})$ with faint optical and near-IR counterparts are likely to be highly obscured type 2 QSOs. This approach has been extensively applied to Spitzer observations of the Chandra Deep Fields $(54 ; 55)$. In these fields, the X-ray coverage is deep and therefore it allows to probe the nature of these mid-IR "excesses". A few of these sources 
have a direct X-ray detection which might indicate that they are obscured sources ${ }^{1}$. The average $\mathrm{X}$-ray properties of a class of sources can be studied using the stacking technique (i.e. summing the signal of different sources). The stacked X-ray spectrum (shown in the left panel of Fig. 5) of mid-IR excesses is compatible with the one of a Compton-thick AGNs (54; 55). Since most of these sources have either a photometric or a measured redshift, it becomes possible to estimate their volume density (see right panel of Fig. 5). This, at the average redshift of these samples $(z \sim 2)$, turns out to be of the same order of that of X-ray detected AGNs. This would imply that the mid-IR selection is a powerful technique to recover the population of Compton-thick objects which is not detected even in the deepest X-ray surveys.

However, the complex selection criteria leave some uncertainty about the true nature of these sources. Indeed, mid-IR excesses might be produced by powerful sturburst galaxies (e.g. 55). In order to remove all uncertainties one would need to obtain an IR and an X-ray spectra for all these sources. Given the large redshift and the number of sources this is not always feasible. At least in the case of HDF-OMD49, a Spitzer object at $\mathrm{z}=2.21$, the IR and X-ray spectra show that this source is very likely a Compton-thick AGN. However, recently (56), using a compbination of IR bands and mid-IR spectroscopy, determined that the majority of the dust obscured galaxies ${ }^{2}$ are dominated by star formation rather than AGN activity. Thus, while mid-IR selection might represent a powerful tool to recover Compton-thick AGNs at large redshifts, it might be that the contamination due to powerful starburst galaxies is at the moment affecting the estimates of the space density of highly-obscured AGNs.

\section{Reflection and Cut-off properties of AGNs}

Reflection and high-energy cut-off are with intrinsic absorption the main spectral properties of AGNs. Studying them is, however, not as simple as studying intrinsic absorption. This is because both reflection and high-energy cut-off produce a weaker spectral signature and with low signal to noise data they tend to be degenerate. The optimum solution would be to use high-quality broad band data which allow to disentangle all three spectral components. Such approach has been recently used for type- 1 and type-2 AGNs by (58) and (59) respectively. Both groups find evidences for spectral cut-offs which are at energies $<300 \mathrm{keV}$ and thus at odds with previous studies in the $1-500 \mathrm{keV}$ range $(60 ; 61)$ which located the cut-off at $>500 \mathrm{keV}$. In most cases a strong reflection component and an Iron line are found, although they appear to be inconsistent with the measured absorbing column densities (59). This might indicate that the absorbing and the reflecting material are not the same or that the absorbing/reflecting material is not uniform (i.e. a clumpy torus model). (62) analyzed a sample of 105 Seyfert objects detected by BeppoSAX and thus with simultaneous broad-band coverage in the $2-100 \mathrm{keV}$ band. He finds a systematic difference between Seyfert 1 and 2 galaxies with Seyfert 1s having a steeper spectrum, a larger reflection component and a lower energy spectral cut-off. This is in agreement with what found for a smaller sample of Swift/BAT sources (31). While the different strengths of the reflection components might be explained by the different viewing angles of the reflecting material in these objects, the different spectral cut-offs

\footnotetext{
${ }^{1}$ Given the signal-to-noise ratios it is very difficult to say whether these sources are simply obscured $\left(\mathrm{N}_{H}>\right.$ $\left.10^{22} \mathrm{~cm}^{-2}\right)$ or are Compton-thick $\left(\mathrm{N}_{H}>10^{24} \mathrm{~cm}^{-2}\right)$.

${ }^{2}$ Dust obscured galaxies are selected in such a way that $90 \%$ of them would meet the selection criteria of (55).
} 

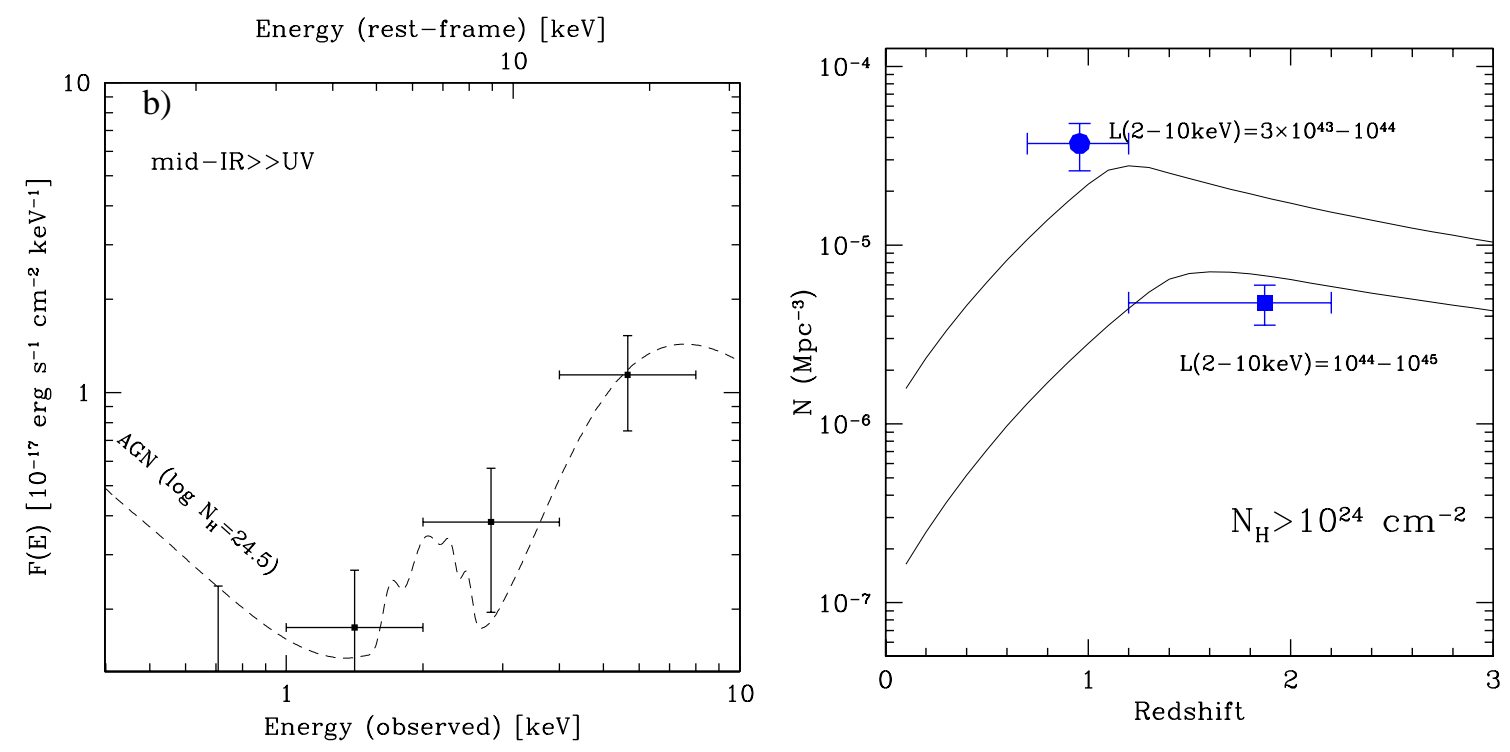

Figure 5: Left: Stacked X-ray spectrum of mid-IR excesses detected in the Chandra deep fields. The spectrum is compatible with the spectrum of a Compton-thick AGN. Adapted from (54). Right:Volume density of mid-IR selected sources in the COSMOS field (57). The solid lines are predictions from the model of (12).

might signal a break down of the AGN unified model. Indeed, in thermal Compton models (e.g. 60) the cut-off energy is related to the thermal energy of the electrons populating the hot corona above the accretion disk and in this case it would imply that this energy is lower in Seyfert 1 with respect Seyfert 2. However, I remark that with low signal to noise spectra is very difficult to disentangle all the different spectral components. The best way would be to select a flux-limited sample of bright Seyfert galaxies for which a $0.1-500 \mathrm{keV}$ data exist. While thanks to XMM-Newton, Swift and INTEGRAL it is possible to assemble high-quality data up to $\sim 200 \mathrm{keV}$, above this energy there are essentially no data. In this framework the detection of Seyfert galaxies above $200 \mathrm{keV}$ (e.g. 63) might play an important role as it would allow to disentangle the spectral cut-off from the other spectral features.

\section{Unveiling the hard X-ray sky: the SIX survey}

INTEGRAL/IBIS and Swift/BAT will likely be able to expose a significant area of the extragalactic sky down to the $0.5 \mathrm{mCrab}^{3}$ flux level. BAT will achieve it thanks to its continuos all-sky coverage, while INTEGRAL will do it through its Key Programme observations (e.g. see the $6 \mathrm{Ms}$ observation of the North Ecliptic Pole). Simbol-X, NuSTAR and NeXT (64; 65; 66, respectively), all focusing hard X-ray telescopes, will be able to sample fluxes of $10^{-13}-10^{-14} \mathrm{erg} \mathrm{cm}^{-2} \mathrm{~s}^{-1}$. Until that time, no other scheduled mission ${ }^{4}$ will probe intermediate fluxes.

\footnotetext{
${ }^{3} \mathrm{As}$ a reference $1 \mathrm{mCrab}$ in the $15-55 \mathrm{keV}$ band is equivalent to $\sim 1.27 \times 10^{-11} \mathrm{erg} \mathrm{cm}^{-2} \mathrm{~s}^{-1}$.

${ }^{4}$ The EXIST mission under study by NASA would probe the $5 \times 10^{-13} \mathrm{erg} \mathrm{cm}^{-2} \mathrm{~s}^{-1}$ fluxes (67).
} 
There is however a way to sample fluxes weaker than the ones sampled by the INTEGRAL/IBIS and Swift/BAT surveys and this is represented by the Swift+INTEGRAL X-ray (SIX) survey (Ajello et al. in prep.). The SIX survey is obtained as a natural combination of both surveys. Indeed, as it is shown in Tab. 2, the performances of both instruments, BAT and IBIS, are similar in terms of sensitivity for deep extragalactic exposures. The main difference is represented by the point spread function (PSF) which is better for INTEGRAL. However, as long as one avoids crowded regions (i.e. the Galactic plane) this issue does not matter. The advantage of the SIX survey is not only that it allows to combine the exposures to obtain a deeper one, but that it smoothes out the relative systematic errors that both instruments have. As a test field to demonstrate the potentiality of the SIX survey we chose the North Ecliptic Pole (NEP) field. This field has been proposed and approved as an INTEGRAL key-programme because of the absence of bright sources which could worsen the sensitivity. At the moment of this writing, the NEP field has been surveyed to $1.7 \mathrm{Ms}$ (of the $6 \mathrm{Ms}$ requested). Using two years of Swift/BAT survey data (68) we get an exposure on the same region of $\sim 4$ Ms. Left panel of Fig. 6 shows the difference between the BAT and the IBIS surveys. Indeed, while IBIS reaches a deeper sensitivity at the centre of the mosaic, the BAT survey has shallower, but much more uniform exposure on the whole area. The SIX survey joins the best of both worlds yielding already in this simple test a sensitivity better than $0.5 \mathrm{mCrab}$. As expected by the combination of two surveys with different systematic errors, the noise is well behaved and the distribution of signal-to-noise ratios is consistent with a normal Gaussian distribution (see right panel of Fig. 6). The number of detected sources above $5 \sigma$ is 18 which is a large number considering that it has been detected in an INTEGRAL mosaic $(\sim 0.5 \mathrm{sr})$ away from the Galactic plane. This test shows the potentiality of this approach fully as it has already produced the most sensitive hard X-ray survey to date. A much better sensitivity is, reasonably, expected when the NEP field will be surveyed by INTEGRAL to the planned $6 \mathrm{Ms}$.

Table 2: Comparison of the BAT and ISGRI instruments. Sensitivities are real in-flight performances and were derived for BAT and ISGRI by Ajello et al. (2008a) and Bassani et al. (2006) respectively.

\begin{tabular}{lcc}
\hline \hline & BAT & ISGRI \\
\hline PSF $(\operatorname{arcmin})$ & 22 & 12 \\
FOV $\left(\mathrm{deg}^{2}\right)$ & 4500 & 400 \\
Energy range $(\mathrm{keV})$ & $15-200$ & $16-300$ \\
$5 \sigma$ Sensitivity in $1 \mathrm{Ms}(\mathrm{mCrab})$ & 0.9 & 0.8 \\
\hline
\end{tabular}

\section{Conclusions}

Thanks to INTEGRAL and Swift the hard X-ray sky looks bright. For the first time in X-ray astronomy, there is an all-sky X-ray selected sample of AGNs which constitutes an unbiased census of AGNs in the local Universe. We have learned that contrary to the expectation, from the unified model, the fraction of absorbed objects, in the local Universe, is $\sim 50 \%$. As deep surveys have shown, the ratio of absorbed sources to the total population is a function of intrinsic luminosity. This has been interpreted as a decrease of the covering factor of the circomnuclear dust as a function of luminosity and finds a natural explanation in the framework of the clumpy torus model which is, as of today, well established. 

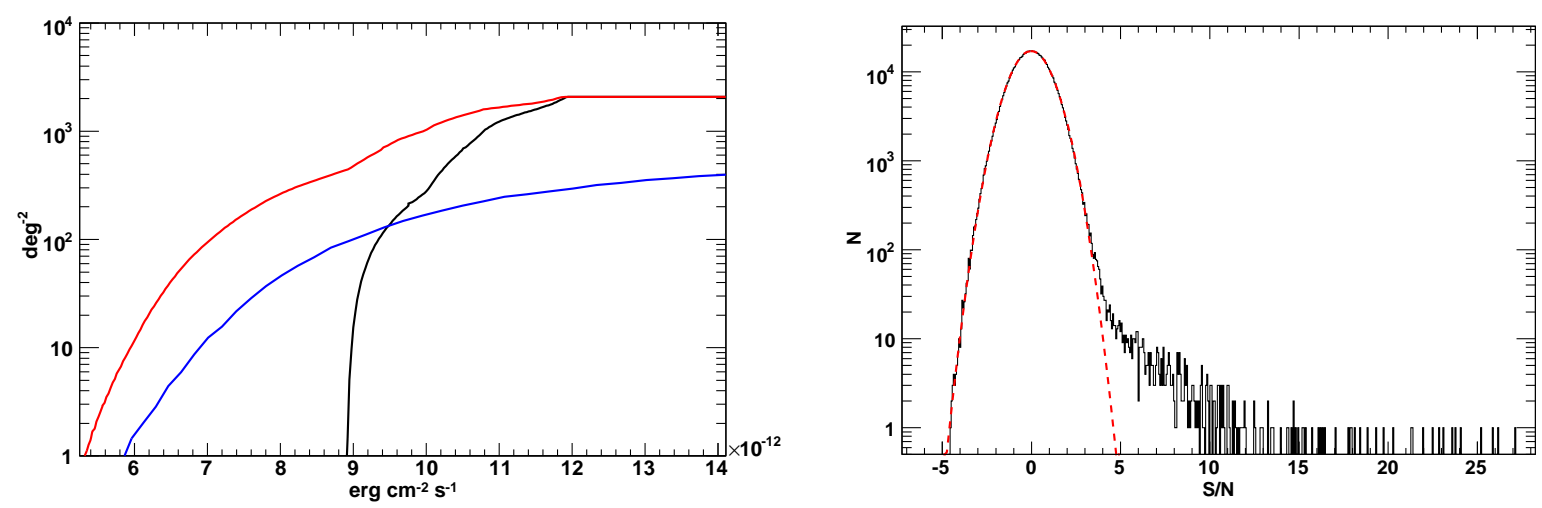

Figure 6: Performances of the SIX survey on a test field. The field chosen is the North Ecliptic Pole (INTEGRAL Key programme) surveyed by INTEGRAL to $1.7 \mathrm{Ms}$. The left panel shows the BAT (black), IBIS (blue) and SIX (red) sky coverages. The limiting sensitivity of the SIX mosaic, in this example, is better than $0.5 \mathrm{mCrab}$. The right panel shows the distribution of pixel significances in the SIX-NEP mosaic. The dashed line is an overlaid Gaussian with $\sigma=1.0$. The long tail at positive significances is given by real sources detected in the mosaic.

Despite their lack, Compton-thick AGNs are still required to explain a substantial part of the Cosmic X-ray background around its peak. One of the main discoveries of INTEGRAL and Swift is to have unveiled a new population of Compton-thick AGNs. These are AGNs with an intense reflection component which seems to come from a material which covers a large solid angle around the central source. The current estimates show that these 'buried' super-massive black hole might be a relevant fraction $(\sim 25 \%)$ of the total population of AGNs, although given their low scattering efficiency their contribution to the CXB spectrum seems small. At the same time, large populations of Compton-thick objects can be recovered detecting the reprocessing, by dust, of UV nuclear emission into IR. The current estimates of their space density at large redshift indicates that Compton-thick AGNs are at least as numerous as the remainder AGN population. The number density of detected Compton-thick objects in the local Universe and in the high-redshift one are in agreement with predictions of population synthesis models showing that we are close to solve the mystery of the generation of the Cosmic X-ray Background. If this view is correct, hard X-ray $(>15 \mathrm{keV}$ ) focusing telescopes will detect many Compton-thick AGNs at intermediate redshifts.

\section{Acknowledgments}

The processing of INTEGRAL data, in the framework of the SIX survey, rests entirely on E. Bottacini whom is here warmly acknowledged. Most of the work presented here comes from my $\mathrm{PhD}$ thesis and I acknowledge all members of the gamma group at MPE as well as the members of the BAT-team at Goddard. 


\section{References}

[1] R. Giacconi, H. Gursky, F. R. Paolini, and B. B. Rossi, Evidence for x Rays From Sources Outside the Solar System, Physical Review Letters 9 (Dec., 1962) 439-443.

[2] H. L. Marshall, H. Tananbaum, Y. Avni, and G. Zamorani, Analysis of complete quasar samples to obtain parameters of luminosity and evolution functions, Astrophys. J. 269 (June, 1983) 35-41.

[3] R. F. Mushotzky, X-ray spectra and time variability of active galactic nuclei, Advances in Space Research 3 (1984) 157-165.

[4] E. L. Wright, J. C. Mather, D. J. Fixsen, A. Kogut, R. A. Shafer, C. L. Bennett, N. W. Boggess, E. S. Cheng, R. F. Silverberg, G. F. Smoot, and R. Weiss, Interpretation of the COBE FIRAS CMBR spectrum, Astrophys. J. 420 (Jan., 1994) 450-456.

[5] G. Setti and L. Woltjer, Active Galactic Nuclei and the spectrum of the X-ray background, A\&A 224 (Oct., 1989) L21-L23.

[6] G. Hasinger, R. Burg, R. Giacconi, G. Hartner, M. Schmidt, J. Trumper, and G. Zamorani, A Deep X-Ray Survey in the Lockman-Hole and the Soft X-Ray N-Log, A\&A 275 (Aug., 1993) $1-+$.

[7] D. M. Alexander, W. N. Brandt, A. E. Hornschemeier, G. P. Garmire, D. P. Schneider, F. E. Bauer, and R. E. Griffiths, The Chandra Deep Field North Survey. VI. The Nature of the Optically Faint X-Ray Source Population, AJ 122 (Nov., 2001) 2156-2176, [arXiv:astro-ph/0107450].

[8] R. Giacconi, A. Zirm, J. Wang, P. Rosati, M. Nonino, P. Tozzi, R. Gilli, V. Mainieri, G. Hasinger, L. Kewley, J. Bergeron, S. Borgani, R. Gilmozzi, N. Grogin, A. Koekemoer, E. Schreier, W. Zheng, and C. Norman, Chandra Deep Field South: The 1 Ms Catalog, APJS 139 (Apr., 2002) 369-410, [arXiv: astro-ph/0112184].

[9] A. Comastri, G. Setti, G. Zamorani, and G. Hasinger, The contribution of AGNs to the X-ray background., A\&A 296 (Apr., 1995) 1-+, [arXiv:astro-ph/9409067].

[10] Y. Ueda, M. Akiyama, K. Ohta, and T. Miyaji, Cosmological Evolution of the Hard X-Ray Active Galactic Nucleus Luminosity Function and the Origin of the Hard X-Ray Background, Astrophys. J. 598 (Dec., 2003) 886-908, [arXiv: astro-ph/0308140].

[11] E. Treister and C. M. Urry, Active Galactic Nuclei Unification and the X-Ray Background, Astrophys. J. 630 (Sept., 2005) 115-121.

[12] R. Gilli, A. Comastri, and G. Hasinger, The synthesis of the cosmic X-ray background in the Chandra and XMM-Newton era, A\&A 463 (Feb., 2007) 79-96, [arXiv:astro-ph/0610939]. 
[13] M. A. Worsley, A. C. Fabian, F. E. Bauer, D. M. Alexander, G. Hasinger, S. Mateos, H. Brunner, W. N. Brandt, and D. P. Schneider, The unresolved hard X-ray background: the missing source population implied by the Chandra and XMM-Newton deep fields, MNRAS 357 (Mar., 2005) 1281-1287, [arXiv:astro-ph/ 0412266 ].

[14] A. Comastri, Compton-Thick AGN: The Dark Side of the X-Ray Background, in Astrophysics and Space Science Library (A. J. Barger, ed.), vol. 308 of Astrophysics and Space Science Library, pp. 245-+, Aug., 2004.

[15] G. Hasinger, T. Miyaji, and M. Schmidt, Luminosity-dependent evolution of soft X-ray selected AGN. New Chandra and XMM-Newton surveys, A\&A 441 (Oct., 2005) 417-434, [arXiv:astro-ph/0506118].

[16] M. Revnivtsev, M. Gilfanov, K. Jahoda, and R. Sunyaev, Intensity of the cosmic X-ray backgound from HEAOl/A2 experiment, A\&A 444 (Dec., 2005) 381-385, [arXiv:astro-ph/0412304].

[17] X. Barcons, S. Mateos, and M. T. Ceballos, On the intensity of the extragalactic X-ray background, MNRAS 316 (July, 2000) L13-L16, [arXiv : ast ro-ph / 0005596 ].

[18] F. Frontera, M. Orlandini, R. Landi, A. Comastri, F. Fiore, G. Setti, L. Amati, E. Costa, N. Masetti, and E. Palazzi, The Cosmic X-Ray Background and the Population of the Most Heavily Obscured AGNs, Astrophys. J. 666 (Sept., 2007) 86-95, [arXiv:astro-ph/0611228].

[19] D. E. Gruber, J. L. Matteson, L. E. Peterson, and G. V. Jung, The Spectrum of Diffuse Cosmic Hard X-Rays Measured with HEAO 1, Astrophys. J. 520 (July, 1999) 124-129, [arXiv:astro-ph/9903492].

[20] P. Ubertini, F. Lebrun, G. Di Cocco, A. Bazzano, A. J. Bird, K. Broenstad, A. Goldwurm, G. La Rosa, C. Labanti, P. Laurent, I. F. Mirabel, E. M. Quadrini, B. Ramsey, V. Reglero, L. Sabau, B. Sacco, R. Staubert, L. Vigroux, M. C. Weisskopf, and A. A. Zdziarski, IBIS: The Imager on-board INTEGRAL, A\&A 411 (Nov., 2003) L131-L139.

[21] S. D. Barthelmy, L. M. Barbier, J. R. Cummings, E. E. Fenimore, N. Gehrels, D. Hullinger, H. A. Krimm, C. B. Markwardt, D. M. Palmer, A. Parsons, G. Sato, M. Suzuki, T. Takahashi, M. Tashiro, and J. Tueller, The Burst Alert Telescope (BAT) on the SWIFT Midex Mission, Space Science Reviews 120 (Oct., 2005) 143-164, [arXiv: astro-ph/ 0507410 ].

[22] E. Churazov, R. Sunyaev, M. Revnivtsev, S. Sazonov, S. Molkov, S. Grebenev, C. Winkler, A. Parmar, A. Bazzano, M. Falanga, A. Gros, F. Lebrun, L. Natalucci, P. Ubertini, J.-P. Roques, L. Bouchet, E. Jourdain, J. Knödlseder, R. Diehl, C. Budtz-Jorgensen, S. Brandt, N. Lund, N. J. Westergaard, A. Neronov, M. Türler, M. Chernyakova, R. Walter, N. Produit, N. Mowlavi, J. M. Mas-Hesse, A. Domingo, N. Gehrels, E. Kuulkers, P. Kretschmar, and M. Schmidt, INTEGRAL observations of the cosmic X-ray background in the 5-100 keV range via occultation by the Earth, A\&A 467 (May, 2007) 529-540, [arXiv:astro-ph/0608250]. 
[23] M. Ajello, J. Greiner, G. Sato, D. R. Willis, G. Kanbach, A. W. Strong, R. Diehl, G. Hasinger, N. Gehrels, C. B. Markwardt, and J. Tueller, Cosmic X-ray background and Earth albedo Spectra with Swift/BAT, ArXiv:0808.3377 (Aug., 2008) [0808 . 3377].

[24] A. Moretti, C. Pagani, G. Cusumano, S. Campana, M. Perri, A. Abbey, M. Ajello, A. P. Beardmore, D. Burrows, G. Chincarini, O. Godet, C. Guidorzi, J. E. Hill, J. Kennea, J. Nousek, J. P. Osborne, and G. Tagliaferri, A new measurement of the cosmic X-ray background, A\&A 493 (Jan., 2009) 501-509, [0811.1444].

[25] F. La Franca, F. Fiore, A. Comastri, G. C. Perola, N. Sacchi, M. Brusa, F. Cocchia, C. Feruglio, G. Matt, C. Vignali, N. Carangelo, P. Ciliegi, A. Lamastra, R. Maiolino, M. Mignoli, S. Molendi, and S. Puccetti, The HELLAS2XMM Survey. VII. The Hard X-Ray Luminosity Function of AGNs up to $z=4$ : More Absorbed AGNs at Low Luminosities and High Redshifts, Astrophys. J. 635 (Dec., 2005) 864-879, [arXiv: astro-ph/0509081].

[26] J. D. Silverman, P. J. Green, W. A. Barkhouse, D.-W. Kim, M. Kim, B. J. Wilkes, R. A. Cameron, G. Hasinger, B. T. Jannuzi, M. G. Smith, P. S. Smith, and H. Tananbaum, The Luminosity Function of X-Ray-selected Active Galactic Nuclei: Evolution of Supermassive Black Holes at High Redshift, Astrophys. J. 679 (May, 2008) 118-139, [0 710.2461$].$

[27] G. Hasinger, Absorption properties and evolution of active galactic nuclei, A\&A 490 (Nov., 2008) 905-922, [0808.0260].

[28] V. Beckmann, S. Soldi, C. R. Shrader, N. Gehrels, and N. Produit, The Hard X-Ray 20-40 keV AGN Luminosity Function, Astrophys. J. 652 (Nov., 2006) 126-135, [arXiv:astro-ph/0606687].

[29] L. Bassani, A. Malizia, J. B. Stephen, and for the INTEGRAL AGN survey team, INTEGRAL/IBIS 20-100 keV Extragalactic survey: an update, astro-ph/0610455 (Oct., 2006) [astro-ph/0610455].

[30] S. Sazonov, M. Revnivtsev, R. Krivonos, E. Churazov, and R. Sunyaev, Hard X-ray luminosity function and absorption distribution of nearby AGN: INTEGRAL all-sky survey, $A \& A 462$ (Jan., 2007) 57-66, [arXiv : astro-ph/ 0608418 ].

[31] M. Ajello, J. Greiner, G. Kanbach, A. Rau, A. W. Strong, and J. A. Kennea, BAT X-Ray Survey. I. Methodology and X-Ray Identification, Astrophys. J. 678 (May, 2008) 102-115, [arXiv:0712.2885].

[32] J. Tueller, R. F. Mushotzky, S. Barthelmy, J. K. Cannizzo, N. Gehrels, C. B. Markwardt, G. K. Skinner, and L. M. Winter, Swift BAT Survey of AGNs, Astrophys. J. 681 (July, 2008) 113-127, [arXiv:0711.4130].

[33] T. Yaqoob, X-Ray Transmission in Cold Matter: Nonrelativistic Corrections for Compton Scattering, Astrophys. J. 479 (Apr., 1997) 184-+. 
[34] I. N. Evans, H. C. Ford, A. L. Kinney, R. R. J. Antonucci, L. Armus, and S. Caganoff, HST imaging of the inner 3 arcseconds of NGC 1068 in the light of forbidden O III 5007 A, APJL 369 (Mar., 1991) L27-L30.

[35] A. Lawrence and M. Elvis, Obscuration and the various kinds of Seyfert galaxies, Astrophys. J. 256 (May, 1982) 410-426.

[36] A. Lawrence, The relative frequency of broad-lined and narrow-lined active galactic nuclei Implications for unified schemes, MNRAS 252 (Oct., 1991) 586-592.

[37] R. Della Ceca, A. Caccianiga, P. Severgnini, T. Maccacaro, H. Brunner, F. J. Carrera, F. Cocchia, S. Mateos, M. J. Page, and J. A. Tedds, The cosmological properties of AGN in the XMM-Newton Hard Bright Survey, A\&A 487 (Aug., 2008) 119-130, [0 805.1919$].$

[38] G. Risaliti, M. Elvis, G. Fabbiano, A. Baldi, A. Zezas, and M. Salvati, Occultation Measurement of the Size of the X-Ray-emitting Region in the Active Galactic Nucleus of NGC 1365, APJL 659 (Apr., 2007) L111-L114, [arXiv: astro-ph/ 0703173$].$

[39] M. Nenkova, M. M. Sirocky, Ž. Ivezić, and M. Elitzur, AGN Dusty Tori. I. Handling of Clumpy Media, Astrophys. J. 685 (Sept., 2008) 147-159, [0806. 0511].

[40] S. F. Hönig and T. Beckert, Active galactic nuclei dust tori at low and high luminosities, MNRAS 380 (Sept., 2007) 1172-1176.

[41] A. Comastri, R. Gilli, C. Vignali, G. Matt, F. Fiore, and K. Iwasawa, Compton Thick AGN in the Suzaku Era, Progress of Theoretical Physics Supplement 169 (2007) 274-277, [arXiv:0704.1253].

[42] Y. Ueda, S. Eguchi, Y. Terashima, R. Mushotzky, J. Tueller, C. Markwardt, N. Gehrels, Y. Hashimoto, and S. Potter, Suzaku Observations of Active Galactic Nuclei Detected in the Swift BAT Survey: Discovery of a "New Type" of Buried Supermassive Black Holes, APJL 664 (Aug., 2007) L79-L82, [arXiv: 0706.1168$].$

[43] G. Risaliti, R. Maiolino, and M. Salvati, The Distribution of Absorbing Column Densities among Seyfert 2 Galaxies, Astrophys. J. 522 (Sept., 1999) 157-164, [arXiv:astro-ph/9902377].

[44] M. Guainazzi, G. Matt, and G. C. Perola, X-ray obscuration and obscured AGN in the local universe, A\&A 444 (Dec., 2005) 119-132, [arXiv : astro-ph/ 0508265 ].

[45] R. Della Ceca, P. Severgnini, A. Caccianiga, A. Comastri, R. Gilli, F. Fiore, E. Piconcelli, P. Malaguti, and C. Vignali, Heavily obscured AGN with BeppoSAX, INTEGRAL, SWIFT, $X M M$ and Chandra: prospects for Simbol-X ., Memorie della Societa Astronomica Italiana 79 (2008) 65-+, [0 709 . 3060$].$

[46] L. M. Winter, R. F. Mushotzky, J. Tueller, and C. Markwardt, X-Ray Properties of an Unbiased Hard X-Ray-detected Sample of Active Galactic Nuclei, Astrophys. J. 674 (Feb., 2008) 686-710, [0711.0019]. 
[47] S. Sazonov, M. Revnivtsev, R. Burenin, E. Churazov, R. Sunyaev, W. R. Forman, and S. S. Murray, Discovery of heavily-obscured AGN among seven INTEGRAL hard X-ray sources observed by Chandra, A\&A 487 (Aug., 2008) 509-517, [0802 . 0928].

[48] S. Paltani, R. Walter, I. M. McHardy, T. Dwelly, C. Steiner, and T. J.-L. Courvoisier, A deep INTEGRAL hard X-ray survey of the 3C 273/Coma region, A\&A 485 (July, 2008) 707-718, [0805.0537].

[49] R. Antonucci, Unified models for active galactic nuclei and quasars, ARAA 31 (1993) 473-521.

[50] C. Simpson, The luminosity dependence of the type 1 active galactic nucleus fraction, MNRAS 360 (June, 2005) 565-572, [arXiv: astro-ph/ 0503500$].$

[51] A. Marconi, G. Risaliti, R. Gilli, L. K. Hunt, R. Maiolino, and M. Salvati, Local supermassive black holes, relics of active galactic nuclei and the X-ray background, MNRAS 351 (June, 2004) 169-185, [arXiv: astro-ph/0311619].

[52] A. Martínez-Sansigre, S. Rawlings, M. Lacy, D. Fadda, F. R. Marleau, C. Simpson, C. J. Willott, and M. J. Jarvis, The obscuration by dust of most of the growth of supermassive black holes, NAT 436 (Aug., 2005) 666-669, [arXiv: astro-ph/ 0505486 ].

[53] J. R. Houck, B. T. Soifer, D. Weedman, S. J. U. Higdon, J. L. Higdon, T. Herter, M. J. I. Brown, A. Dey, B. T. Jannuzi, E. Le Floc'h, M. Rieke, L. Armus, V. Charmandaris, B. R. Brandl, and H. I. Teplitz, Spectroscopic Redshifts to $z>2$ for Optically Obscured Sources Discovered with the Spitzer Space Telescope, APJL 622 (Apr., 2005) L105-L108, [arXiv:astro-ph/0502216].

[54] E. Daddi, D. M. Alexander, M. Dickinson, R. Gilli, A. Renzini, D. Elbaz, A. Cimatti, R. Chary, D. Frayer, F. E. Bauer, W. N. Brandt, M. Giavalisco, N. A. Grogin, M. Huynh, J. Kurk, M. Mignoli, G. Morrison, A. Pope, and S. Ravindranath, Multiwavelength Study of Massive Galaxies at z 2. II. Widespread Compton-thick Active Galactic Nuclei and the Concurrent Growth of Black Holes and Bulges, Astrophys. J. 670 (Nov., 2007) 173-189, [arXiv: 0705.2832].

[55] F. Fiore, A. Grazian, P. Santini, S. Puccetti, M. Brusa, C. Feruglio, A. Fontana, E. Giallongo, A. Comastri, C. Gruppioni, F. Pozzi, G. Zamorani, and C. Vignali, Unveiling Obscured Accretion in the Chandra Deep Field-South, Astrophys. J. 672 (Jan., 2008) 94-101, [arXiv:0705.2864].

[56] A. Pope, R. S. Bussmann, A. Dey, N. Meger, D. M. Alexander, M. Brodwin, R.-R. Chary, M. E. Dickinson, D. T. Frayer, T. R. Greve, M. Huynh, L. Lin, G. Morrison, D. Scott, and C.-H. Yan, The Nature of Faint Spitzer-selected Dust-obscured Galaxies, Astrophys. J. 689 (Dec., 2008) 127-133, [0808.2816].

[57] F. Fiore, S. Puccetti, M. Brusa, M. Salvato, G. Zamorani, T. Aldcroft, H. Ausse, H. Brunner, P. Capak, N. Cappelluti, F. Civano, A. Comastri, M. Elvis, C. Feruglio, A. Finoguenov, 
A. Fruscione, R. Gilli, G. Hasinger, A. Koekemoer, J. Kartaltepe, O. Ilbert, C. Impey, E. Le Floc'h, S. Lilly, V. Mainieri, A. Martinez-Sansigre, H. J. McCracken, N. Menci, A. Merloni, T. Miyaji, D. B. Sanders, M. Sargent, E. Schinnerer, N. Scoville, J. Silverman, V. Smolcic, A. Steffen, P. Santini, Y. Taniguchi, D. Thompson, J. R. Trump, C. Vignali, M. Urry, and L. Yan, Chasing highly obscured QSOs in the COSMOS field, ArXiv e-prints (Oct., 2008) [0810.0720].

[58] F. Panessa, L. Bassani, A. de Rosa, A. J. Bird, A. J. Dean, M. Fiocchi, A. Malizia, M. Molina, P. Ubertini, and R. Walter, The broad-band XMM-Newton and INTEGRAL spectra of bright type 1 Seyfert galaxies, A\&A 483 (May, 2008) 151-160, [0803.0896].

[59] A. de Rosa, L. Bassani, P. Ubertini, F. Panessa, A. Malizia, A. J. Dean, and R. Walter, An $X$-ray view of absorbed INTEGRAL AGN, A\&A 483 (June, 2008) 749-758, [0801 . 4675].

[60] A. A. Zdziarski, W. N. Johnson, C. Done, D. Smith, and K. McNaron-Brown, The average $X$-ray/gamma-ray spectra of Seyfert galaxies from GINGA and OSSE and the origin of the cosmic X-ray background, APJL 438 (Jan., 1995) L63-L66.

[61] D. Gondek, A. A. Zdziarski, W. N. Johnson, I. M. George, K. McNaron-Brown, P. Magdziarz, D. Smith, and D. E. Gruber, The average X-ray/gamma-ray spectrum of radio-quiet Seyfert 1s, MNRAS 282 (Sept., 1996) 646-652, [arXiv:astro-ph/9607016].

[62] M. Dadina, Seyfert galaxies in the local Universe $(z<0.1)$ : the average X-ray spectrum as seen by BeppoSAX, A\&A 485 (July, 2008) 417-424, [0 801 . 4338].

[63] L. Bouchet, E. Jourdain, J.-P. Roques, A. Strong, R. Diehl, F. Lebrun, and R. Terrier, INTEGRAL SPI All-Sky View in Soft Gamma Rays: A Study of Point-Source and Galactic Diffuse Emission, Astrophys. J. 679 (June, 2008) 1315-1326, [0801 .2086].

[64] P. Ferrando, M. Arnaud, U. Briel, O. Citterio, R. Clédassou, P. Duchon, F. Fiore, P. Giommi, A. Goldwurm, G. Hasinger, E. Kendziorra, P. Laurent, F. Lebrun, O. Limousin, G. Malaguti, S. Mereghetti, G. Micela, G. Pareschi, Y. Rio, J. P. Roques, L. Strüder, and G. Tagliaferri, Simbol-X: mission overview, in Society of Photo-Optical Instrumentation Engineers (SPIE) Conference Series, vol. 6266 of Presented at the Society of Photo-Optical Instrumentation Engineers (SPIE) Conference, July, 2006.

[65] F. A. Harrison, F. E. Christensen, W. Craig, C. Hailey, W. Baumgartner, C. M. H. Chen, J. Chonko, W. R. Cook, J. Koglin, K.-K. Madsen, M. Pivavoroff, S. Boggs, and D. Smith, Development of the HEFT and NuSTAR focusing telescopes, Experimental Astronomy 20 (Dec., 2005) 131-137.

[66] T. Takahashi, R. Kelley, K. Mitsuda, H. Kunieda, R. Petre, N. White, T. Dotani, R. Fujimoto, Y. Fukazawa, K. Hayashida, M. Ishida, Y. Ishisaki, M. Kokubun, K. Makishima, K. Koyama, G. M. Madejski, K. Mori, R. Mushotzky, K. Nakazawa, Y. Ogasaka, T. Ohashi, M. Ozaki, H. Tajima, M. Tashiro, Y. Terada, H. Tsunemi, T. G. Tsuru, Y. Ueda, N. Yamasaki, and 
S. Watanabe, The NeXT Mission, in Society of Photo-Optical Instrumentation Engineers (SPIE) Conference Series, vol. 7011 of Presented at the Society of Photo-Optical Instrumentation Engineers (SPIE) Conference, Aug., 2008.

[67] J. E. Grindlay, EXIST: All-sky hard X-ray imaging and spectral-temporal survey for black holes [review article], New Astronomy Review 49 (Nov., 2005) 436-439, [arXiv:astro-ph/0512657].

[68] M. Ajello, P. Rebusco, N. Cappelluti, O. Reimer, H. Böhringer, J. Greiner, N. Gehrels, J. Tueller, and A. Moretti, Galaxy Clusters in the Swift/burst Alert Telescope Era: Hard $X$-Rays in the Intracluster Medium, Astrophys. J. 690 (Jan., 2009) 367-388, [0809.0006]. 